\title{
LOS DERECHOS FUNDAMENTALES EN LA ENCRUCIJADA DE LA LUCHA CONTRA EL TERRORISMO YIHADISTA. LO QUE EL CONSTITUCIONALISMO Y EL DERECHO DE LA UNIÓN EUROPEA PUEDEN OFRECER EN COMÚN ${ }^{1}$
}

\author{
ROSARIO SERRA CRISTÓBAL \\ Profesora Titular de Derecho Constitucional \\ Universidad de Valencia
}

SUMARIO

I. Los derechos fundamentales en la encrucijada entre seguridad y libertad. II. La respuesta de la UE en la lucha contra el terrorismo III. La jurisprudencia del Tribunal de Justicia en el ámbito de la lucha contra el terrorismo internacional y la protección de los derechos fundamentales IV. Principios compartidos en el seno de la UE que deben presidir la lucha contra el terrorismo yihadista.

\section{LOS DERECHOS FUNDAMENTALES EN LA ENCRUCIJADA ENTRE SEGURIDAD Y LIBERTAD}

Los atentados terroristas perpetrados por el ISIS en Paris en 2015 (enero y noviembre), en Copenhague en 2015 y en Bruselas en 2016 han puesto de nuevo en alerta a los Estados europeos y acentuado un sentimiento de vulnerabilidad que ya existía desde los atentados de las Torres Gemelas ${ }^{2}$ y los que le siguieron en Madrid (2004) y Londres (2005), entre otros. La sensación de una amenaza global que tiene puesto su punto de mira en Europa ha agudizado la necesidad de dar

1 La presente investigación se integra dentro del proyecto de $\mathrm{I}+\mathrm{D}$ «Seguridad global y derechos fundamentales: la protección contra las amenazas y la garantía de las libertades», del Ministerio de Economía y Copetitividad (Ref. DER2015-65288-R).

2 Revenga SÁnchez, M. (ed.), Terrorismo y Derecho bajo la estela del 11 de septiembre, Valencia: Tirant lo Blanch, 2014. 
una respuesta supranacional al fenómeno del terrorismo yihadista. Pero, como sabemos, esta respuesta no resulta innocua para los derechos fundamentales.

Por un lado, el terrorismo se considera una de las más flagrantes violaciones de los derechos humanos y constituye una amenaza para la paz y la democracia. Pero al mismo tiempo, muchas de las medidas adoptadas para combatirlo, — no solo para la persecución de los autores, sino también las puestas en marcha con carácter preventivo-, han dejado dudosos ejemplos de respeto por los derechos humanos ${ }^{3}$.

Nos encontramos ante el binomio seguridad-libertad, que es el lugar común al que nos conducen las respuestas de las sociedades democráticas ante los atentados terroristas. Pese a que, como se ha señalado, es cierto que «entre libertad y seguridad no hay ninguna tensión, y no la hay porque la seguridad es el elemento constitutivo de la libertad» ${ }^{4}$, la verdad es que ese no es el sentir mayoritario. Precisamente la salvaguarda de la seguridad nacional se ha convertido en argumento para adoptar medidas que parecen más encaminadas a limitar esa libertad que a asegurarla. El objetivo de este trabajo es defender que, en esa encrucijada de la lucha antiyihadista, contamos con principios e instrumentos, que son compartidos en el constitucionalismo europeo y que constituyen el fundamento de la UE, que pueden ayudar a que el peso de las políticas antiterroristas en Europa no recaiga exclusivamente en la defensa de la seguridad a costa de un sacrificio desproporcionado de nuestras libertades.

\section{LA RESPUESTA DE LA UE EN LA LUCHA CONTRA EL TERRORISMO}

Comencemos recordando dos postulados que a veces se olvidan. Los Estados miembros de la UE se encuentran vinculados individualmente por el Derecho internacional de los derechos humanos y la Unión se fundamenta en los derechos humanos tal y como están recogidos en el CEDH. Por lo tanto, en la lucha contra el terrorismo yihadista, el respeto a los mismos debe ser una premisa. Así, partimos de que la réplica de los gobiernos a dicho fenómeno, que ya no puede ser exclusivamente doméstica y necesita del establecimiento de estrategias comunes, ha de tener como límite la consideración de los derechos fundamentales 5 .

3 Masferrer, A. y Walker, C. (eds.): Counter-Terrorism, Human Rights and the Rule of Law, Crossing Legal Boundaries in Defence of the State, Elgar, 2013.

4 Perez Royo, J., «La democracia frente al terrorismo global», Pérez Royo (dir.) y Carrasco DuRÁN (coord.), Terrorismo, democracia y seguridad, en perspectiva constitucional, Barcelona: Marcial Pons, 2010, p. 7.

5 Las posibles contradicciones entre la política antiterrorista de la UE y los derechos de los individuos han sido puestas de manifiesto especialmente el Parlamento Europeo y el Tribunal de Justicia. Recuérdese, por ejemplo la Resolución del Parlamento, de 15 de diciembre de 2005, sobre la presunta utilización de países europeos, por parte de la CIA, para el transporte y la detención ilegal de presos, (DO C 286 E de 23.11.2006, p. 509); la Decisión, de 18 de enero de 2006, por la que se constituye una comisión temporal 
A pesar de que en el ámbito de la UE la responsabilidad principal de diseñar el sistema legal en materia de seguridad nacional y de su aplicación a nivel nacional recae en cada Estado miembro (art. 72 TFUE), la Unión se ha embarcado en la armonización de los sistemas jurídicos con el fin de lograr una mayor eficacia en la lucha contra el terrorismo (Art. 29 del Tratado de la UE) ${ }^{6}$, al igual que en una cooperación policial y judicial en materia penal ${ }^{7}$, regulada en el Título $\mathrm{V}$ del Tratado de TFUE, cuyo art. 83 se refiere explícitamente al terrorismo como forma grave de delincuencia. Esa cooperación se ve reforzada con el art 222 del TFUE, que estipula una cláusula de solidaridad. Ésta exige la acción conjunta de todos los países de la UE en caso de que uno de ellos sea objeto de un ataque terrorista $^{8}$. Y a ello hay que añadir el Dispositivo Integrado de Respuesta Política de la UE a las Crisis (DIRPC) adoptado en 2013.

La estrategia terrorista de la UE se ha sustanciado principalmente a través de la Política Exterior y de Seguridad común (PESC), el antiguo segundo pilar que sigue rigiéndose por las reglas de cooperación intergubernamental, aunque también se ha apoyado en la política comunitaria de interior y en el Espacio Europeo de Seguridad, Libertad y Justicia, donde el papel del Parlamento y de la Comisión son más notorios. Por lo tanto, la política de la UE en materia terrorista se articula sobre la base de los principios básicos de la armonización de legislaciones y el reconocimiento mutuo de resoluciones judiciales, pero la cuestión de la seguridad sigue siendo en último extremo una cuestión fundamentalmente estatal,

sobre la presunta utilización de países europeos por la CIA para el transporte y la detención ilegal de presos (DO C 287 E de 24.11.2006, p. 159); o la Resolución, de 6 de julio de 2006, sobre la supuesta utilización de países europeos por la CIA para el transporte y la detención ilegal de presos.

Asimismo, el Parlamento Europeo mostró su preocupación por la privacidad de los ciudadanos en relación al Programa de Seguimiento de la Financiación del Terrorismo al que luego haremos referencia (TFTP UE-EE.UU.).

6 Un desarrollo de esta cooperación puede encontrarse, entre otros, en el art. 4 de la Posición común del Consejo de 27 de diciembre de 2001, sobre the application of specific measures to combat terrorism (aplicación de medidas especiales para combatir el terrorismo) (2001/931/CFSP); la Decisión del Consejo de 28 de noviembre de 2002, que establee un mecanismo para evaluar los sistemas legales y su implementación a nivel nacional en la lucha contra el terrorismo (2002/996/JHA). Véase también la Decisión del Consejo 2003/48/ JHA, de 19 de diciembre de 2002, sobre the implementation of specific measures for police and judicial cooperation to combat terrorism in accordance with Article 4 of Common Position 2001/931/CFSP; y la Decisión Marco del Consejo de 13 de junio de 2002, sobre the European arrest warrant and the surrender procedures between Member States (2002/584/JHA).

7 En dicho ámbito se adoptaron la Decisión Marco 2008/675/JAI del Consejo, de 24 de julio de 2008, relativa a la consideración de las resoluciones condenatorias entre los Estados miembros de la Unión Europea con motivo de un nuevo proceso penal; la Decisión Marco 2008/315/JAI, de 26 de febrero de 2009, relativa a la organización y al contenido del intercambio de información de los registros de antecedentes penales entre Estados miembros; y la Decisión 2008/633/JAI sobre el acceso para consultar el Sistema de Información de Visados (VIS) por las autoridades designadas de los Estados miembros y por Europol, con fines de prevención, detección e investigación de delitos de terrorismo y otros delitos graves.

Las Decisiones citadas se implementaron en España con la aprobación de la Ley Orgánica 7/2014, sobre intercambio de antecedentes penales y consideración de resoluciones judiciales en la Unión Europea.

8 En este ámbito el Consejo adoptó la Decisión, de 24 de junio de 2014, relativa a las modalidades de aplicación por la Unión de la cláusula de solidaridad (2014/415/UE). 
donde escasean los controles comunitarios ${ }^{9}$. Así, por poner un ejemplo, incluso el intercambio de información que se ha acordado en esa lucha antiterrorista no es una obligación cuando un Estado considere que su divulgación puede ser contraria a los intereses esenciales de su propia seguridad ${ }^{10}$. De hecho, la respuesta a los ataques yihadistas perpetrados en Europa en los últimos años han dado lugar a una respuesta decidida y protagonizada de forma principal por los Estados que han visto en riesgo su propia seguridad.

La cooperación entre los Estados de la UE se hizo más evidente después de los ataques del 11-S, la verdadera fecha de lanzamiento de una política común y estructurada de lucha contra el terrorismo. Desde entonces, la cooperación europea ha hecho grandes progresos en este campo. Al igual que en su día hicieron los responsables de la seguridad exterior, elaborando una Estrategia Europea de Seguridad (2003) para reivindicar a la UE como un actor global de seguridad en el entorno post 11-S, los responsables de la cooperación en la materia de justicia e interior decidieron en 2009 aprovechar el lanzamiento del Programa de Estocolmo para elaborar una Estrategia de Seguridad Interior (ESI). La ESI (adoptada el 23 de febrero de 2010) analiza las amenazas y desafíos a los que se enfrentan los Estados de la UE, entre los que incluye el terrorismo, y enumera los principios y líneas estratégicas necesarias para elaborar un modelo de seguridad europeo ${ }^{11}$, o más bien para establecer un modelo de cooperación intergubernamental en algunas materias de seguridad interior. En ella se indica que la UE «debe consolidar un modelo de seguridad, basado en los principios y valores de la Unión: el respeto a los derechos humanos y las libertades fundamentales, el Estado de Derecho, la democracia, el diálogo, la tolerancia, la transparencia y la solidaridad» ${ }^{12}$.

Las políticas de la UE de lucha contra el terrorismo han tratado de no olvidar el respeto por los derechos fundamentales, al menos en sus declaraciones formales. Así, ya en la Decisión marco del Consejo, de 13 de junio de 2002, sobre la lucha contra el terrorismo se establecía que: «Nada en la presente Decisión mar-

9 Sobre el origen de la seguridad como una materia muy ligada al ámbito de lo estatal puede verse el trabajo: Ridaura Martínez, J.: «La seguridad ciudadana como función del Estado», Estudios de Deusto, 62:2, 2014, pp. 319-346.

10 Artículo 8 de la Decisión, de 24 de junio de 2014 relativa a las modalidades de aplicación por la Unión de la cláusula de solidaridad (2014/415/UE).

11 Sin embargo, no define claramente qué ámbitos corresponden a las autoridades nacionales y cuáles a la Unión.

12 Como parte de la general del Programa de la UE «Seguridad y defensa de las libertades «, se adoptó un programa específico para la Prevención, preparación y gestión de las consecuencias del terrorismo y otros riesgos de seguridad relacionados para el período 2007-2013. La Decisión del Consejo 2007-2013, de 12 febrero 2007, establecía para el período de 2007 a 2013, parte del Programa general de seguridad y defensa de las libertades, Programa específico de prevención, preparación y gestión de las consecuencias del terrorismo y de otros riesgos relacionados con la seguridad (2007/124 / CE, Euratom). Véase también, la Comunicación de la Comisión al Parlamento Europeo y al Consejo «La Estrategia de Seguridad Interior de la UE en acción cinco medidas para una Europa más segura», que propone acciones para la implementación de la estrategia durante el periodo 2011-14 (COM/2010/0673 final). 
co podrá interpretarse como un intento de reducir u obstaculizar derechos o libertades fundamentales tales como el derecho de huelga, la libertad de reunión, de asociación o de expresión, incluido el derecho de toda persona a fundar y afiliarse a sindicatos con otros para la protección de sus intereses y el correspondiente derecho a manifestarse» (considerando n.10).

Desafortunadamente, a parte de ese considerando, ninguna otra disposición de dicha Decisión marco establece acciones concretas para evitar daños a los derechos fundamentales en caso de medidas que se adopten para luchar contra futuras amenazas terroristas. En la misma línea, en documento de debate del Consejo sobre la «Estrategia de la UE de lucha contra el Terrorismo» (23 de mayo de 2012), se indicaba que la comunidad internacional necesita pautas para asegurar que tenemos capacidad de lucha contra el terrorismo, y recordaba que el estado de derecho y los derechos humanos son inherentes a los esfuerzos para combatir el terrorismo (p. 10) ${ }^{13}$.

Los atentados de París de 2015 y de Bruselas en 2016 han empujado a los Estados de la Unión Europea y a sus instituciones a reevaluar su política de seguridad, y proponer nuevas iniciativas para reforzar la lucha antiterrorista, en concreto en el establecimiento de controles sistemáticos de fronteras, control de armas y registro de pasajeros. Precisamente esos atentados aceleraron la adopción de la Directiva 2016/680, de 27 de abril, sobre el tratamiento de datos por las autoridades en el ámbito de la prevención y persecución del crimen ${ }^{14}$, junto con el nuevo Reglamento 2016/679 de Protección de Datos ${ }^{15}$. Igualmente, la Comisión Europea presentó en diciembre de 2015 un Proyecto de Directiva de lucha antiterrorista $^{16}$, que vendría a sustituir a la Decisión marco 2001/475/JHA sobre la misma materia, aunque este texto todavía está en fase de debate.

En la lucha antiterrorista, la necesidad de cooperación en inteligencia ha sido una cuestión recurrente en la UE, porque la prevención de nuevos ataques terroristas puede reforzarse, entre otras cosas, mediante el establecimiento de programas de intercambio de información sobre delincuentes, fronteras, transacciones financieras, etc.

13 Consejo de la UE, 9990/12.

14 Directive (EU) 2016/680 of the European Parliament and of the Council of 27 April 2016 on the protection of natural persons with regard to the processing of personal data by competent authorities for the purposes of the prevention, investigation, detection or prosecution of criminal offences or the execution of criminal penalties, and on the free movement of such data, and repealing Council Framework Decision 2008/977/JHA. Entró en vigor el 5 de Mayo de 2016, aunque los Estados miembros disponen hasta el 6 de Mayo de 2108 para implementarla.

15 Regulation (EU) 2016/679 of the European Parliament and of the Council of 27 April 2016 on the protection of natural persons with regard to the processing of personal data and on the free movement of such data, and repealing Directive 95/46/EC (General Data Protection Regulation). Entró en vigor el 24 de Mayo 2016, pero empezará aplicarse a partir del 25 de Mayo de 2018.

16 COM (2015) 625 final, 2015/0281 (COD) Brussels, 2.12.2015. 
Con este propósito, en el área Schengen se aprobó la Decisión Marco del Consejo 2006/960/JAI, que estableció un sistema simplificado de intercambio de información e inteligencia entre los servicios de seguridad de los Estados miembros de la Unión Europea que, no obstante, no impone imperativamente el intercambio de ese tipo de datos, pero sí facilita su transmisión entre los servicios de seguridad. También se creó una herramienta que podría ser muy eficaz sobre el papel pero ha sido infrautilizada: el sistema de información Schengen, una base de datos conectada con la policía en la que introducir alertas que saltan automáticamente cuando un viajero buscado en un país se dirige a cualquier aeropuerto de la zona de libre circulación. Los datos a los que nos estamos refiriendo tienen cauces para ser intercambiados a través de Europol y Eurojust, pero los centros de inteligencia estatales tradicionalmente han tendido a trabajar en clave doméstica y han recelado de compartir toda la información.

La nueva Directiva 2016/680 sobre tratamiento de datos en la prevención y persecución del crimen, mencionada arriba, abre un nuevo escenario más reforzado en el ámbito del intercambio de datos entre Estados. La Directiva se aplicará tanto al procesamiento transfronterizo de datos personales, como al tratamiento de datos personales por parte de las autoridades policiales y judiciales a nivel puramente estatal. Ha de tenerse en cuenta que la anterior Decisión Marco 2008, que queda sustituida por la Directiva, sólo cubría el intercambio transfronterizo de datos. La nueva Directiva hace hincapié en la creciente necesidad de intercambiar datos para la prevención y la lucha contra la delincuencia transnacional y el terrorismo. Pero, igualmente subraya la obligación de garantizar el cumplimiento de los principios básicos que regulan la protección de datos personales (Considerando n. ${ }^{\circ}$ ). De hecho, establece normas mínimas que han de respetarse en el tratamiento de datos dentro de cada Estado miembro, se establecen derechos concretos y se fijan limites en el flujo de datos.

Entre las medidas de intercambio de información, una cuestión muy debatida ha sido una propuesta, que llevaba estancada desde 2013, de establecer un Registro de pasajeros aéreos que podría compartirse entre los Estados de la UE —el PNR (Personal Name Records)_. El Parlamento Europeo mostró reiteradamente su preocupación por el daño que podría generar para la privacidad de los viajeros $^{17}$.

A pesar de ello, tras los atentados de Paris de noviembre de 2015, en el Consejo de Ministros de Interior de 4 de diciembre de ese año, se dio luz verde a la aprobación del citado Registro, que quedó finalmente regulado por la Directiva reguladora del uso del PNR para la prevención, investigación y persecución del

17 Así, en abril de 2013, el Parlamento Europeo rechazó una propuesta para crear y utilizar, a nivel de la UE, un Registro PNR por sus dudas sobre su necesidad y sus preocupaciones sobre la privacidad y los datos de los ciudadanos europeos. 
terrorismo y otros crímenes graves, aprobada el 21 de abril de $2016^{18}$. La Directiva será implementada por los Estados en dos años. Se aplicará a los vuelos que tienen origen o destino fuera de la UE, pero los Estados miembros también podría extenderlo a los domésticos («dentro de la UE»). Ello obligará, a partir de su trasposición en dos años, a la transmisión a las autoridades de una serie de datos de los pasajeros de vuelos (nombre, dirección, itinerario, modo de pago, tarjetas bancarias utilizadas, números de teléfono, información sobre equipaje, fechas de viajes, frecuencia con que vuela...). La cuestión está en cómo se va a hacer con el máximo respeto a la privacidad de los europeos. Con ese fin, entre otras previsiones, la Directiva establece que tales transferencias sólo se harán «siguiendo la regla de caso por caso» y exclusivamente para los fines específicos de «prevenir, detectar, investigar y procesar los delitos de terrorismo o delitos graves».

En esa misma línea, en mayo de 2015, el Consejo y el Parlamento Europeo adoptaron nuevas normas destinadas a prevenir el blanqueo de capitales y la financiación del terrorismo (Directiva (UE) 2015/849 del Parlamento Europeo y del Consejo, de 20 de mayo de 2015, relativa a la prevención de la utilización del sistema financiero para el blanqueo de capitales o la financiación del terrorismo), medidas que también incluyen el intercambio de información ${ }^{1920}$. Y el 11 de febrero de 2016 el Consejo de la Unión acogió con satisfacción el Plan de la Comisión de 2 de febrero de 2016 para reforzar la lucha contra la financiación del terrorismo $^{21}$ instándole, en todo caso, a la revisión de la normativa sobre los instrumentos de prepago, blanqueo de capitales y las monedas virtuales, y animando a la cooperación e intercambio de información entre los países a estos efectos.

Estas últimas medidas guardan relación con el Terrorism Finance Tracking Program (EU-US TFTP), un acuerdo firmado entre la UE y Estados Unidos en 2001, y renovado en varias ocasiones, para compartir datos entre las autoridades de dichos estados con el fin de luchar contra el terrorismo y en concreto contra su financiación. Este programa permite el intercambio del código SWIFT, que se usa para las transacciones monetarias realizadas entre bancos, y que es particularmente útil para realizar el seguimiento de aquéllas que se hacen por vía telemática. Desde el principio, el TFTP ha generado información

18 Directive regulating the use of Passenger Name Record (PNR) data in the EU for the prevention, detection, investigation and prosecution of terrorist offences and serious crime.

19 Con anterioridad a la Directiva mencionada, se adoptaron otras. En concreto, en relación al intercambio de información, ha de citarse la Cuarta Directiva contra el blanqueo de capitales y Reglamento (UE) 2015/847 del Parlamento Europeo y del Consejo, de 20 de mayo de 2015, relativo a la información que acompaña a las transferencias de fondos.

20 El terrorismo yihadista se financia con dinero que procede de distintas fuentes: secuestros de europeos, contrabando, tráfico de refugiados causado por el conflicto de Siria, etc. Ello supone el movimiento de mucho dinero que se transfiere en parte a través de cuentas bancarias. Cierto es que cada vez más cada grupo terrorista en cada zona del mundo organiza sus propios medios de financiación. A este respecto, puede verse un interesante artículo de Loreta Napoleoni: «Así se financia el terror yihadista», El País, 16 de noviembre de 2015 .

21 Comisión Europea Documento 5782/16 + ADD1. 
significativa para los servicios de inteligencia europeos y ha sido útil en la investigación de numerosas amenazas terroristas, incluida la relación de nacionales de la UE con los grupos islamistas extremistas en Siria. Ha sido una herramienta clave para detectar el movimiento de fondos por parte de terroristas a través de transacciones financieras o la identificación de las redes terroristas y colaboradores. Sin embargo, también se han planteado muchas preguntas acerca de si se protegen debidamente los derechos fundamentales y en concreto aquellos relacionados con la privacidad.

Asimismo, en relación con el intercambio de datos con terceros países, procede recordar que recientemente se ha producido una renovación del Acuerdo entre la UE y Estados Unidos conocido como «Safe Harbour» por el «EU-US Privacy Shield» de febrero de 2016, precisamente por haber sido declarado inválido aquel marco previo por el Tribunal de Luxemburgo en el caso Schrems ${ }^{22}$. Nos remitimos a lo que a este respecto analizaremos en el siguiente apartado.

\section{LA JURISPRUDENCIA DEL TRIBUNAL DE JUSTICIA EN EL ÁMBITO DE LA LUCHA CONTRA EL TERRORISMO INTERNACIONAL Y LA PROTECCIÓN DE LOS DERECHOS FUNDAMENTALES}

Entre los instrumentos que puede servir de límite a los excesos de los gobiernos y legisladores en la lucha antiterrorista se encuentra el control judicial. Tanto en la esfera nacional como de la UE los tribunales desempeñan un papel de fiscalización que puede convertirse en una de las mejores garantías para la protección de los derechos fundamentales. En la UE ese papel lo desempeña el Tribunal de Justicia.

Una de las dificultades con la que se enfrenta el Tribunal en materia de terrorismo reside en que sólo tiene competencia indirecta y limitada sobre la Política Exterior y de Seguridad Común. Sin embargo, desde 1 de diciembre de 2014, tiene plena jurisdicción sobre Cooperación Policial y Judicial (Intercambio de información, la cooperación antiterrorista, extradiciones/detención, lucha contra el crimen organizado...) Aun con esas limitaciones, el Tribunal de Luxemburgo ha tomado desde hace años una postura a favor de la promoción de los derechos en la lucha contra el terrorismo. Y ello ha sido así pese a que el Tribunal de Justicia es un «recién llegado» al campo de la lucha contra el terrorismo y otros asuntos de seguridad nacional, si lo comparamos con el TEDH o los tribunales nacionales. El Tribunal de Estrasburgo y otros tribunales constitucionales o superiores nacionales se han visto en la obligación de pronunciar- 
se en situaciones de crisis de seguridad nacional desde ya hace algunas décadas. Los recursos interpuestos ante el Tribunal de Justicia son más recientes y, debido a las características especiales de la distribución de poderes (supranacionales e internacional) de la UE, su papel como órgano de vigilancia de los derechos humanos se ha convertido en un reto difícil cuando se trata del terrorismo internacional.

En todo caso, en dicho ámbito el Tribunal de Justicia siempre ha invocado el legado común europeo en materia de derechos humanos y el imperio de la ley. En el caso Öcalan (C-229/05, 18 de enero de 2007, Sala Primera), la Corte recordó: «La Comunidad Europea es una comunidad basada en el Estado de Derecho cuyas instituciones están sujetas a revisión judicial de la compatibilidad de su actuación con el Tratado y con los principios generales del Derecho que forman parte los derechos fundamentales. Por lo tanto, las personas tienen derecho a la tutela judicial efectiva de los derechos que les confiere el ordenamiento jurídico de la Comunidad, y el derecho a esa protección es uno de los principios generales del Derecho que resultan de las tradiciones constitucionales comunes a los Estados miembros [...] y en el Europeo Convención sobre los Derechos Humanos».

El Tribunal reconoció en el caso citado que, si bien la normativa nacional puede establecer medidas restrictivas dirigidas contra determinadas personas y entidades con el fin de luchar contra el terrorismo, cabe establecer limitaciones a esas facultades de los Estados Miembros.

Otra de las dificultades que enfrenta el TJCE es no solo la necesidad de cumplir con su función de vigilancia de los derechos humanos con respecto a la legislación de la UE (y no siempre con plena jurisdicción sobre todas las cuestiones), sino el hacerlo, al mismo tiempo, sin dejar de lado las normas comunes derivadas de las Constituciones, la jurisprudencia CEDH y los acuerdos y obligaciones internacionales de la UE. El caso Kadi (TJCE 2008) ${ }^{23}$ es un ejemplo de constitucionalismo en acción en un marco transnacional. El asunto Kadi es quizás el caso del TJCE más visible e interesante de los últimos años con respecto a las relaciones exteriores y la seguridad. El Tribunal esencialmente tenía que decidir si una Resolución del Consejo de Seguridad de la ONU debía tener prioridad sobre la legislación comunitaria. Consideró que la ejecución de dicha Resolución de la ONU a través del Reglamento n. ${ }^{\circ}$ 188/2002 constituía una violación clara y evidente de los derechos humanos, y que, como éstos deben ser respetados por la UE, no cabía el cumplimiento de aquélla. Como sostiene Kokott y Sobotta ${ }^{24}$, el enfoque de la Corte en este asunto podría ser caracterizado como una variación del llamado concepto «Solange». Este concepto fue desarrollado por el Tribunal

23 Yassin Abdullab Kadi and Al Barakaat International Foundation v. Council of the European Union and Commission of the European Communities (TJUE. Asuntos acumulados C-402/05 P and C-415/05 P, 3 Septiembre 2008).

24 Кокотт J. Sовота, Ch., The Kadi Case-Constitutional Core Values and International Law-Finding the Balance?, European Journal of International Law, 32:4, 2012, p. 1016. 
Constitucional alemán ${ }^{25}$ e igualmente aplicado por el $\mathrm{TEDH}^{26}$, que también ha podido ser testigo de la interacción entre los diferentes organismos nacionales y supranacionales.

Kadi fue identificado como un posible defensor de Al-Qaida por el Consejo de Seguridad de la ONU, se le incluyó en una lista de terroristas y fue sancionado. La UE transpuso esa sanción de la ONU a través del citado Reglamento $188 / 2002$, que imponía medidas restrictivas contra personas y entidades vinculadas a Bin Laden, la red Al-Qaida y los talibanes, incluidas en una lista adjunta. El Reglamento fue impugnado por Kadi ante los tribunales de la UE. Kadi alegó que no había sido informado de los motivos de su inclusión en la lista de personas y entidades sujetas a las sanciones establecidas. Por lo tanto, no había tenido la posibilidad de presentar un recurso judicial contra dichos motivos y, en consecuencia, su derecho a ser oído, así como su derecho a la tutela judicial efectiva habían sido vulnerados. El Tribunal de Justicia examinó la legalidad de la normativa de la UE, que trasponía la Resolución de la ONU, con el argumento de que la protección de los derechos fundamentales forma parte de los fundamentos del orden jurídico de la UE. De acuerdo con este principio, todas las medidas de la UE deben ser compatibles con los derechos fundamentales, de lo que resultaba la aplicación preferente del Derecho de la Unión frente a la ejecución de compromisos internacionales.

El caso Kadi ha sido la base para nuevas decisiones como las adoptadas en el asunto Kadi $\mathrm{II}^{27}$, y en otros como el asunto Omar Mohammed Othbam de 11 de junio de $2009^{28}$, el caso Al-Aqsa, de 15 de noviembre de 201229, o el Abdulabsit Abdulrabim de 14 de enero de $2015^{30}$. En el primero de los asuntos, el recurso también se dirigió contra la aplicación del Reglamento C n. ${ }^{\circ}$ 188/2002, que imponía medidas restrictivas contra personas y entidades vinculadas al terrorismo yihadista. El recurrente alegó que las instituciones comunitarias no le habían informado de los datos utilizados en su contra y que justificaban las medidas sancionadoras que se le habían aplicado (incautación de bienes y congelación de

25 El concepto «Solange» proviene del caso Solange I (1974). Aquí, the Bundesverfassungsgericht decidió analizar su derecho a revisar la acción de la UE en relación con el Derecho nacional en materia de derechos fundamentales cuando existe una deficiente protección de los mismos en el ámbito de la Unión Europea.

26 El Tribunal Europeo de Derechos Humanos siguió un razonamiento similar en su sentencia del Bósforo (2005), donde optó por abstenerse de ejercer el control con respecto a los actos de la UE. La sentencia del Bósforo fue visto por muchos analistas como una oferta de compromiso, invitando a los tribunales de Luxemburgo para continuar su jurisprudencia sobre derechos humanos y la preparación del terreno para la UE para adherirse al CEDH.

27 Asunto T-85/09 Kadi v. Commission, 2010. Asunto confirmado por el Tribunal de Luxemburgo en su sentencia de 18 July 2013, European Commission, UE Council and United Kingdom v. Yassin Abdullah Kadi (Asuntos acumulados C-584/10P, C-593/10P and C595/10P).

28 Asunto Omar Mohammed Othham contra el Consejo y la Comisión, de 11 de junio de 2009, resuelto por el Tribunal de Primera Instancia.

29 Asunto C/539/10 P.

30 Sentencia del Tribunal General de 14 de enero de 2015, (asunto T-127/09 RENV). 
fondos económicos), ni se le había permitido presentar pruebas en su defensa. El Tribunal de Primera Instancia reconoció tales vulneraciones y consideró nulo el referido Reglamento en lo que afectaba al recurrente ${ }^{31}$.

Además, también en relación a esa inclusión de personas en las listas de terroristas (listas negras terroristas), el TJUE consideró que «los nombres de personas, grupos o entidades se pueden mantener en la lista sólo si el Consejo revisa periódicamente su situación. Todos estos asuntos deben estar abiertos a la revisión judicial» $»^{32}$.

La cuestión que se planteó el caso Kadi y en los siguientes sigue estando vigente y es extrapolable a otras esferas. Así, por ejemplo, cabe preguntarse si, ante las respuestas globales o internacionales que puedan adoptarse en la lucha contra el terrorismo, los Estados miembros de la UE pueden y deben verificar la ejecución de tales medidas de conformidad con sus normas Constitucionales y también con los Tratados supranacionales que exigen el respeto de los derechos fundamentales. Por supuesto, la respuesta debe ser afirmativa por todo lo que venimos defendiendo en este trabajo y por el principio de favor libertatis, debiendo optarse siempre por la interpretación que ofrezca mayores garantías a los derechos.

Por otro lado, el Tribunal de Justicia se ha pronunciado asimismo sobre otras medidas adoptadas en la lucha contra el terrorismo y los delitos transnacionales. El Tribunal de Justicia emitió su parecer en supuestos relativos a pruebas secretas (asunto C-27/09 P República Francesa v. OMPI, 201133). A este respecto, consideró que un individuo debe, en todo caso, ser informado como mínimo de los motivos que conducen a su consideración como presunto terrorista (caso de $Z Z v$. Secretario de Estado del Ministerio del Interior, 2013) ${ }^{34}$.

Igualmente, se ha posicionado en defensa de los derechos a la intimidad y privacidad de los ciudadanos europeos en la lucha contra el terrorismo. Así lo hizo, por ejemplo, cuando resolvió un recurso presentado por el Parlamento Europeo sobre la compartición de datos personales de los usuarios de líneas aéreas en el asunto $P N R^{35}$ al que hacíamos referencia más arriba. El Tribunal anuló tanto la Decisión del Consejo que autorizaba la firma de un tratado con Estados Unidos para que las compañías aéreas europeas le trasfirieran los datos personales de sus viajeros, como la Decisión de la Comisión Europea que consideraba que, en ese

31 Hay otros casos pendientes ante el Tribunal de Justicia sobre el mantenimiento de una empresa o individuo en la lista de presuntos colaboradores con el terrorismo (Ese el es caso del asunto National Iranian Oil Company (NIOC) v. Consejo (Asunto C-440/14P), (Las conclusiones del abogado general fueron presentadas el 3 septiembre de 2015); id. en petición de decisión prejudicial por el Landgericht Essen (Alemania), de enero de 2015, sobre la inclusión de Liberation Tigers of Tamil Eelam en la lista de entidades terroristas.

32 en su sentencia de 18 July 2013, European Commission, UE Council and United Kingdom v. Yassin Abdullah Kadi (Asuntos acumulados C-584/10P, C-593/10P and C595/10P).

33 Asunto C-27/09.

34 Asunto C-300/11.

35 STJCE (Gran Sala) Personal Name Records, asuntos acumulados C-371/04 y C-318/04, de 30 de agosto de 2006. 
proceso, Estados Unidos protegía los datos de manera adecuada. Para el Tribunal los estándares europeos en materia de protección de datos no quedaban suficientemente salvaguardados por aquel estado.

En la misma línea de proteger la trasferencia de datos de los ciudadanos europeos, el TJUE invalidó, en el asunto Digital Rights Ireland y Seitlinger y otros $(2014)^{36}$, la Directiva 2006/24/CE del Parlamento Europeo y del Consejo, de 15 de marzo de 2006, sobre la conservación de datos generados o tratados en relación con la prestación de servicios de comunicaciones electrónicas de acceso público o de redes públicas de comunicaciones. Esta Directiva permitía la conservación de los datos de tráfico y localización, y de identificación del abonado o usuario para fines de prevención y persecución de delitos graves como la delincuencia organizada y el terrorismo. Para el Tribunal, aunque dicha conservación de datos para su posible trasmisión a las autoridades nacionales responde a un objetivo de interés general, —la seguridad pública—, el legislador de la Unión sobrepasó los límites que exige el principio de proporcionalidad. El modo en que la Directiva regulaba la conservación de datos suponía una injerencia amplia y especialmente grave en los derechos fundamentales, siendo necesaria una regulación que garantice que dicha intervención se limite efectivamente a lo estrictamente necesario.

Igualmente, el Tribunal invalidó el 6 de octubre de $2015^{37}$ la decisión Safe Harbour (Puerto seguro) de la Comisión, aprobada en 2000, que facilitaba la transferencia de datos personales desde países miembros de la UE hacia Estados Unidos, decisión que supuso una victoria para la privacidad de los ciudadanos europeos. Gracias a aquel acuerdo las compañías adheridas al mismo, como Facebook, Google, Amazon o Apple, eran reconocidas como puertos seguros y podían transferir bajo su amparo información entre Estados Unidos y la UE. La normativa europea de protección de datos sólo permite transferir datos a países que cuenten con un nivel adecuado de seguridad, premisa que, a los ojos del Tribunal, no cumplía Estados Unidos. Para el TJUE el Acuerdo permitía que prevaleciera la seguridad nacional o el interés público sobre los derechos fundamentales a la intimidad y la protección de datos, sin permitir que los ciudadanos tuviesen mecanismos para proteger con efectividad esos derechos. Además, el Acuerdo no ofrecía a los Estados miembros un margen suficiente para suspender las transferencias en caso de que apreciasen una vulneración de los derechos de los ciudadanos europeos ${ }^{38}$. El nuevo Acuerdo que se ha adoptado en 2016, el EU-US Privacy Shield, y que vienen a reemplazar al Safe Harbour, establece mayores obligaciones

36 Sentencia Tribunal de Justicia de la Unión Europea, Gran Sala, de 8 de abril de 2014, Asuntos acumulados C-293/12 y C-594/12.

37 Asunto C-362/14 Maximillian Schrems v. Data Protection Commissioner.

38 Lo deseable sería que esta doctrina del TJUE se incluyera en el Reglamento de protección de datos que se está elaborando y sobre cuyos contenidos generales se alcanzó un acuerdo en el ámbito de la UE el 16 de junio de 2015 . 
a las empresas estadounidenses respecto a la protección de datos personales de los europeos, al igual que una mayor cooperación con las autoridades europeas de protección de datos. Este Acuerdo incluye ciertas garantías adicionales cuando las autoridades públicas accedan a los datos personales transferidos para fines de interés general, estableciéndose condiciones, limitaciones y un sistema de supervisión, y evitando el acceso generalizado.

\section{PRINCIPIOS COMPARTIDOS EN EL SENO DE LA UE QUE DEBEN PRESIDIR LA LUCHA CONTRA EL TERRORISMO YIHADISTA}

En definitiva, de lo abordado más arriba se deduce que hay principios compartidos por los Estados de la UE y fundamento de ésta que no pueden olvidarse en el marco de la lucha contra el terrorismo yihadista. Son principios que han de aplicarse a la persecución de los delitos de terrorismo y a las medidas preventivas llevadas a cabo por los servicios de inteligencia y de defensa que buscan evitar nuevos ataques terroristas.

El primer principio que ha de recordarse es que los derechos y libertades son una prioridad. Los derechos fundamentales son una parte esencial de un sistema jurídico democrático y ocupan una posición privilegiada dentro de ella. Veíamos más arriba como la UE establece en sus Tratados que ésta se fundamenta en el respeto a los mismos. Esto da lugar a la prohibición de su infracción, salvo cuando esté justificado por razones de interés general y, en todo caso, la limitación ha de ser proporcional al interés general perseguido.

En consecuencia, es imprescindible determinar los riesgos y amenazas a la seguridad que pueden justificar suficientemente restricciones a los derechos fundamentales y en qué condiciones. No podemos negar que muchas veces la imposición de restricciones a los derechos es necesaria en la búsqueda de objetivos legítimos como la seguridad nacional, la seguridad pública, o la batalla contra el terrorismo internacional. Incluso, en ocasiones, son el resultado de las obligaciones derivadas de las Resoluciones del Consejo de Seguridad de la ONU o de otros compromisos internacionales. Sin embargo, hemos de analizar si la interferencia en los derechos fundamentales es necesaria y si es proporcionada al fin legítimo perseguido, y si los motivos invocados por las autoridades nacionales para justificarla son "pertinentes», es decir, si es posible recurrir a una medida alternativa que causaría menos daño al derecho fundamental en cuestión, para cumplir con el mismo objetivo.

En segundo lugar, incluso en circunstancias excepcionales, hay un nivel mínimo de respeto de los derechos fundamentales que deber ser infranqueable. Los atentados terroristas han conducido a varios países a la declaración del estado de emergencia, con considerables limitaciones de los derechos de la ciudadanía. En las Constituciones, el estado de emergencia refiere a una situación por la cual se 
confieren a las autoridades poderes ampliados (pero también limitados) para hacer frente a una amenaza a la estabilidad de las instituciones públicas, los derechos de los ciudadanos o intereses generales. Pero, la declaración de emergencia debiera requerir la concurrencia de determinadas circunstancias, la intervención del Parlamento, su limitación temporal y, más importante aún, la identificación de aquellos derechos que podrían ser limitados y el alcance de dicha limitación.

Se ha demostrado que no todos los textos constitucionales están preparados para emergencias como el terrorismo internacional. Nos encontramos ante un tipo de terrorismo de tipo difuso, es ejecutado por sujetos que se pueden encontrar en cualquier parte del mundo y utiliza nuevos mecanismos para actuar ${ }^{39}$. Tal vez, la Constitución española es de las pocas que ofrece una suspensión excepcional de los derechos en el marco de la lucha antiterrorista, aunque es una suspensión pensada solo para personas determinadas, en relación a investigaciones de esta naturaleza, y debe ir acompañada de una orden judicial y del resto de garantías constitucionales (art. 55.2 CE). Se trata de una disposición destinada inicialmente a combatir el terrorismo interno (ETA), pero obviamente puede aplicarse a cualquier otro tipo de terrorismo. De hecho, esa posibilidad de limitar derechos en investigaciones de delitos terroristas se incluye en la nueva redacción dada a la Ley de Enjuiciamiento Criminal (LECrim) por la Ley Orgánica 13/2015, de 5 de octubre, de modificación de ésta para el fortalecimiento de las medidas de investigación tecnológica ${ }^{40}$.

En tercer lugar, el respeto por el Estado de Derecho constituye otra lección nuclear extraída del constitucionalismo y compartida por el ordenamiento de la UE. Y, por supuesto, es un principio nuclear para los derechos fundamentales. Cualquier acción en materia de derechos debe ser ejecutada al amparo de la ley. Eso implica que toda actuación contra el terrorismo que afecte a los derechos humanos tiene que ser adoptada de conformidad con la ley. El imperio de la ley sirve para evaluar la legitimidad de determinadas medidas adoptadas ante la amenaza real de ataques terroristas, y para observar si en su ejecución se han respetado los requisitos legales (por ejemplo, la exigencia de una orden judicial cuando la ley así lo requiere). En esta línea, en España el preámbulo de la Ley

39 De Vergottini, G., Más allá del diálogo entre tribunales. Comparación y relación entre jurisdicciones, Madrid: Civitas, 2010, p. 24.

40 La ley incluye numerosas disposiciones que establecen excepciones o límites mayores a los derechos fundamentales cuando se están llevando a cabo investigaciones en materia de terrorismo. Teniendo como límite el marco que establece el art. 55.2 CE respecto de la detención y las libertades domiciliaria y de secreto de comunicaciones, la LECrim regula con detenimiento la interceptación de las comunicaciones escritas, telefónicas y telemáticas (SMS, correos electrónicos, redes sociales y agentes encubiertos en la red, información contenida en USBs, control remoto de ordenadores ...) Así, por ejemplo, y sin entrar en detalles, cuando se trata de la averiguación de delitos relacionados con la actuación de elementos terroristas, y en caso de urgencia, no es imprescindible la autorización previa del juez para intervenir comunicaciones, aunque sí la comunicación posterior a éste. La normativa española, con sus deficiencias, es un ejemplo de que es necesario poner de relieve los límites fundamentales del poder del Estado en situaciones de emergencia o excepcionalidad. Ésta es la única manera de garantizar una protección efectiva y eficaz de los derechos humanos y evitar abusos. 
Orgánica 2/2015 de modificación del Código Penal en materia de terrorismo recuerda que «Estas nuevas amenazas deben, por tanto, ser combatidas con la herramienta más eficaz que los demócratas pueden emplear frente al fanatismo totalitario de los terroristas: la ley».

Desde el punto de vista de la legalidad, la existencia de un marco jurídico supranacional previene también del abuso del término «terrorismo» y dificulta la adopción de políticas que vulneren los derechos humanos ${ }^{41}$.

En cuarto lugar, la eficacia del Estado de Derecho está ligado al principio de la rendición de cuentas de las autoridades públicas (tanto judiciales como políticas). La rendición de cuentas judicial permite a los tribunales comprobar si las acciones de lucha contra el terrorismo respetan los derechos humanos y otras reglas del Derecho. La responsabilidad política se refiere más a la obligación de explicar las razones que hay detrás de las acciones y políticas de un Estado.

Ambos tipos de rendición de cuentas son, a veces, difíciles de garantizar en lo que se refiere a la lucha contra el terrorismo debido a la opacidad de las acciones de los servicios de inteligencia. El trabajo de los servicios de inteligencia y los instrumentos antiterroristas utilizados requiere del secreto. Las Constituciones no obligan a que las cuestiones de seguridad nacional deban ser publicitadas. De hecho, el secreto permite que ciertas fuentes, misiones, eventos o identidades no sean dados a conocer a los que podrían hacer uso de esa información con el fin de actuar en contra de los intereses y la seguridad del Estado. El problema es que, si no se nos informa, es difícil supervisar la actividad del Estado y exigir a los funcionarios el rendimiento de cuentas. Encontrar una manera de hacer que estas actividades queden sujetas a controles democráticos ordinarios constituye un reto. Para solucionar esto, los principios democráticos comunes al Constitucionalismo europeo y al Derecho comunitario, como la transparencia y la rendición de cuentas, deben ponerse efectivamente en práctica ${ }^{42}$.

En quinto lugar, los derechos fundamentales imponen obligaciones a los Estados, que son tanto negativas como positivas. El derecho constitucional y europeo a la seguridad da derecho a los ciudadanos a ser protegidos, ya que las personas no pueden proporcionarse tal seguridad por sí mismas. Frente a la amenaza del terrorismo, las personas pueden esperar que se adopten ciertas políticas

41 Así, en el ámbito de la UE, la Posición común 2001/931/PESC, sobre la aplicación de medidas específicas de lucha contra el terrorismo, la Decisión Marco 2002/475/JAI y la Decisión que la modifica (2008/91/JAI) conminaron a los países dela UE a acercar sus legislaciones e introducir unas penas mínimas en relación con los delitos de terrorismo. Estos textos definen los delitos de terrorismo, así como los delitos relativos a grupos terroristas o ligados a actividades terroristas. Igualmente, se adoptó un listado común de organizaciones terroristas. En septiembre de 2010 Hamás recurrió ante el Tribunal General su presencia continuada en la lista de terroristas de la UE. En diciembre de 2014, el Tribunal General anuló, por motivos de procedimiento, la Decisión del Consejo de mantener a Hamás en la lista. En su reunión de Asuntos Exteriores del 19 de enero de 2015, el Consejo decidió recurrir la sentencia del Tribunal General en el asunto Consejo/Hamás.

42 Cole, D., Fabbrini, F., Verdaschi, A. (eds.), Secrecy, National Security and the Vindication of Constitutional Law, Northampton: Edward Elgar Publisher, 2013. 
y que los poderes públicos actúen para sentirse más seguros. Como se ha visto más arriba, para responder a este deber, se han adoptado diferentes estrategias de pugna contra el terrorismo, tanto en el plano nacional como en el supranacional. Sin embargo, de nuevo hay que subrayar que la seguridad no debe lograrse a costa de restricciones injustificadas de otras libertades. Debemos seguir siendo conscientes del hecho de que la seguridad significa proteger la libertad de las personas, de tal manera que todo el mundo pueda disfrutar de sus derechos sin sentirse amenazado, pero también sin el temor de ser sometido continuamente a limitaciones en sus libertades, especialmente cuando estas limitaciones pueden ser desproporcionadas. Se requiere de un enfoque de la seguridad diferente.

Por último, la sexta lección comúnmente asumida es que el alcance de los derechos fundamentales ya no es una cuestión reservada a la soberanía nacional. Los derechos humanos van más allá de las fronteras del Estado y su protección constituye una cuestión supranacional. En el caso de la amenaza del terrorismo, ya que ésta se ha convertido en un problema mundial, se requiere una respuesta también supranacional que no pierda la perspectiva del respeto de los derechos humanos. El TJUE, al igual que el Tribunal de Estrasburgo, ofrece parámetros para la protección de los derechos en la encrucijada de la lucha antiterrorista. Los casos que se han citado establecen normas básicas de protección de los derechos, impone límites a las autoridades y buscan un equilibrio entre los derechos y la seguridad.

La tensión existente entre las políticas de seguridad y los derechos humanos seguirá existiendo siempre, pero se trata de encontrar un equilibrio. Una respuesta al terrorismo yihadista que sea sensible al respeto a los derechos humanos no parece ser incompatible con la salvaguardia de la seguridad. Porque, si se admite que cualquier cosa vale para hacer frente al terrorismo, podría acabar atentándose contra los mismos valores democráticos y de Derecho que se pretenden proteger en las Constituciones y en la UE.

TITLE: Fundamental Rights at the Crossroads of the Fight against Jihadist Terrorism. What Constitutionalism and European Union Law may offer in common

ABSTRACT: At the crossroads of the fight against Jihadist terrorism, many rights and freedoms result limited. This dissertation analyses whether limits could be settle, from the EU framework, on the States' autonomy to decide on national security policies. The essay argues that principles and tools exist, that are common to both the European constitutionalism and to the EU' basis, and could contribute to balance the counter-terrorism fight strategies in favour of national security but without a disproportionate damage to our liberties.

RESUMEN: En la encrucijada de la lucha contra el terrorismo yibadista, numerosos derechos y libertades se han visto lamentablemente restringidos. Lo que se analiza en este trabajo es si, desde el marco de la UE, cabe establecer límites a esa capacidad individual de los Estados de adoptar decisiones en materia de seguridad nacional. Se defiende que contamos con principios e instrumentos, que son compartidos en el constitucionalismo europeo y que constituyen el fundamento de la UE, que pueden ayudar a que el peso de las políticas antiterro- 
ristas en Europa no recaiga exclusivamente en la defensa de la seguridad a costa de un sacrificio desproporcionado de nuestras libertades.

KEY WORDS: terrorism, fundamental rights, counter-terrorism, security, cooperation within the EU.

PALABRAs ClAVE: terrorismo, derechos fundamentales, lucha antiterrorista, seguridad, cooperación en la $U E$.

FECHA DE RECEPCIÓN: 21.02.2016

FECHA DE ACEPTACIÓN: 27.07.2016 
